\title{
Pulmonary Involvement in Secondary Syphilis
}

\author{
Ruben Benainous ${ }^{1}$, Mohanad Alunji ${ }^{2}$, Pierre-Yves Brillet ${ }^{3}$, Robin Dhote $^{1}$ \\ ${ }^{1}$ Sorbonne Paris Nord University, Service de Médecine Interne, Hôpital Avicenne, Bobigny, France \\ ${ }^{2}$ Sorbonne Paris Nord University, Service de Dermatologie, Hôpital Avicenne, Bobigny, France \\ ${ }^{3}$ Sorbonne Paris Nord University, Service de Radiologie, Hôpital Avicenne, Bobigny, France
}

Received: 15/03/2021

Accepted: $21 / 06 / 2021$

Published: 19/07/2021

How to cite this article: Benainous R, Alunji M, Brillet PY, Dhote R. Pulmonary involvement in secondary syphilis. EJCRIM 2021;8:

doi:10.12890/2021_002487.

Conflicts of Interests: The authors declare there are no competing interests.

This article is licensed under a Commons Attribution Non-Commercial 4.0 License

\section{ABSTRACT}

In secondary syphilis, Treponema pallidum can spread to the lungs. A new case is described of a patient with multiple excavated subpleural pulmonary nodules, a rare localization of secondary syphilis. Despite the numerous pulmonary samples analysed in the literature, T. pallidum is rarely visualised directly on bronchial fibroscopy or biopsy. The diagnosis of secondary syphilis is suspected from historical and physical findings and confirmed by high values obtained from non-treponemal tests.

\section{LEARNING POINTS}

- The clinical and radiological presentation of pulmonary involvement in secondary syphilis is non-specific, described as solitary or multiple subpleural nodular opacities that are rarely infiltrating.

- We confirm here that for this localization, biopsy of these nodules is not necessary for diagnosis.

\section{KEYWORDS}

Syphilis, pulmonary nodule, bronchial fibroscopy, Treponema pallidum

\section{INTRODUCTION}

In secondary syphilis, Treponema pallidum spreads to multiple organs, including the lungs. We here describe a new case of this rare localisation and discuss the diagnostic methods involved.

\section{CASE DESCRIPTION}

A 46-year-old man was hospitalised for weight loss of $7 \mathrm{~kg}$ in 1 month; a dry cough without fever had appeared a few days previously. His blood pressure was normal, and saturation at rest was $98 \%$. Clinical examination revealed a maculopapular, itchy and slightly scaly rash that had started on his back and spread to the chest and limbs (Fig. 1a), together with several cervical lymphadenopathies and a genital ulceration scar. Cardiac and thoracic examinations were normal.

Laboratory analyses indicated normal haemoglobin (14.8 g/dl) and neutrophil (11.2 g/l) counts, and elevated levels of platelets (421 g/l) and C-reactive protein $(35.7 \mathrm{mg} / \mathrm{l})$. ASAT, ALAT, alkaline phosphatase, $\mathrm{\gamma}$-glutamyl transpeptidase, creatinine, LDH and urinalysis results were normal. Serological tests for HIV, hepatitis C and hepatitis B proved negative, as did the test for tuberculosis (IFN- $y$ release assay).

Asymptomatic chorioretinitis was diagnosed in both eyes. An analysis of the CSF was normal. A chest CT revealed several subpleural nodules at the bases of the lungs, the larger being hypodense (Fig. 1b,c). The VDRL test (titre 1/8) and ELISA anti-treponema antibody index (titre 25 , normal <1) were positive. A skin biopsy revealed T. pallidum within a pleomorphic inflammation of the dermal layer. 
A diagnosis of secondary syphilis was made, the primary infection having been asymptomatic. The patient was treated with intravenous penicillin $\mathrm{G}$ for 14 days.
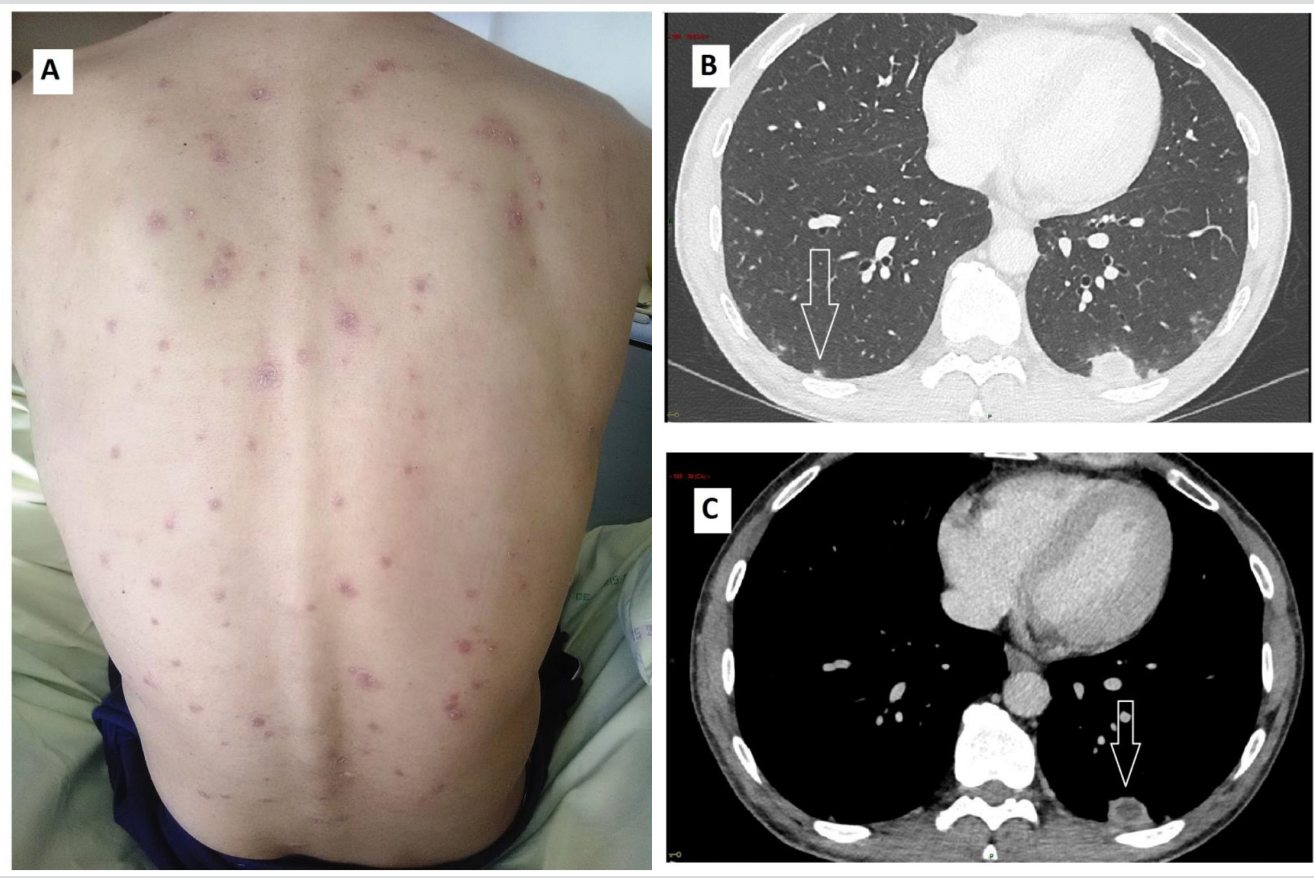

Figure 1 Disseminated maculopapular scaly rash (A). Chest CT: Multiple bi-basal subpleural nodules (arrows) (B), hypodense on the mediastinal window (C)

\section{DISCUSSION}

Pulmonary involvement in syphilis has rarely been reported in the past, with recent reports occurring mainly in the context of secondary syphilis $^{[1]}$.

The clinical and radiological presentation of this pulmonary involvement is non-specific. Patients are usually asymptomatic or complain of mild symptoms ${ }^{[1]}$. Any respiratory symptoms (mainly cough) should trigger a chest x-ray and CT scan. Pulmonary lesions may appear as subpleural nodular opacities that are solitary or multiple and rarely infiltrating, pleural effusion or lymphadenopathy ${ }^{[1-3]}$. Even if no lesion is specific to syphilis, a chest CT scan is indicated to exclude other diseases.

Despite the analysis of numerous pulmonary samples, the direct visualisation of T. pallidum is rarely achieved in the context of syphilis. In only one case was it found in a pleural sample ${ }^{[2]}$, and it was detected in two bronchoalveolar lavage specimens, once using PCR ${ }^{[4]}$ and once by immunohistochemistry ${ }^{[5]}$. Darkfield microscopy of bronchial washing specimens was negative in all cases ${ }^{[4]}$. Rare cases of a bronchial biopsy or open lung biopsy were always negative for T. pallidum, and revealed only peribronchovascular infiltrate and non-specific granuloma ${ }^{[4-6]}$.

In this context, criteria for clinical diagnosis have been proposed by Coleman et al. to define pulmonary syphilis and do not include bronchoscopy and lung biopsy ${ }^{[7]}$ : (a) historical and physical findings typical of secondary syphilis; (b) serological test results positive for syphilis; (c) pulmonary abnormalities seen radiographically, with or without associated symptoms or signs; (d) the exclusion of other forms of pulmonary disease, when possible, in light of the findings of serological tests, sputum smears and cultures and sputum cytology; and (e) a response to anti-syphilis therapy by the signs determined radiologically.

However, unusual clinical and radiological findings (particularly in the context of immune depression or HIV co-infection) require a more aggressive diagnostic strategy in order to exclude other, specific diseases (other infections, neoplastic disease): bronchoalveolar lavage and lung biopsy.

In all the cases referred to above, the diagnosis of secondary syphilis was suspected from historical and physical findings and confirmed by the high values obtained from non-treponemal tests. 


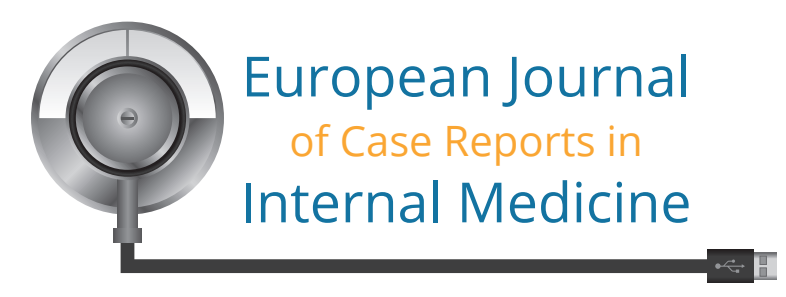

\section{REFERENCES}

Ohta A, Furusyo N, Kishihara Y, Eiraku K, Murata M, Kainuma M, et al. Secondary syphilis with pulmonary involvement. Intern Med 2018;57:121-126. Zaharopoulos P, Wong J. Cytologic diagnosis of syphilitic pleuritis: a case report. Diagn Cytopathol 1997;16:35-38.

Elzouki AN, Al-Kawaaz M, Tafesh Z. Secondary syphilis with pleural effusion: case report and literature review. Case Rep Infect Dis 2012;2012:409896.

David G, Perpoint T, Boibieux A, Pialat JB, Salord H, Devouassoux M, et al. Secondary pulmonary syphilis: report of a likely case and literature review. Clin Infect Dis 2006;42:e11-15.

5. Jeny F, Fargelat A, Laurent-Roussel S, Nault JC, Dupin N, Grange P, et al. Pulmonary consolidations due to secondary syphilis with positive bronchial washing immunohistochemistry. Am J Respir Crit Care Med 2016;193:1061-1062.

6. Soares Souza Jr A, Soares Souza A, Zanetti G, Marchiori E. A skin rash with multiple pulmonary nodules. Eur Respir Rev 2015;24:682-683.

7. Coleman DL, McPhee SJ, Ross TF, Naughton JL. Secondary syphilis with pulmonary involvement. West J Med 1983;138:875-878. 